\title{
The characteristics of the consistency function of the general mean method
}

\author{
Yutaka Kato and Masanori Ozawa \\ College of Engineering, Hosei University \\ 3-7-2, Kajino-cho, Koganei, 'Tokyo 184-0002, Japan \\ Faculty of Science and Technology, Keio University \\ 3-14-1, Hiyoshi, Kohoku-ku, Yokohama 223-8522, Japan \\ ozawa@ae.keio.ac.jp
}

\begin{abstract}
In this study, we consider the estimated weight by the general mean method which has one parameter. The estimated weight is one of the type of mean that the parameter is defined, for instance arithmetic mean, harmonic mean or geometric mean. To find the best parameter, we simulate some cases of the errors included in the pairwise comparison matrix. As a result, the weight vector with the negative parameter is suitable.
\end{abstract}

Key words AHP, simulation, general mean, harmonic mean

\section{The consistency function}

In AHP, to measure a consistency of the matrix of pairwise comparisons, the function of the maximum eigenvalue and the Saaty's consistency function are used well in many papers.

The function of the maximum eigenvalue is given by the next relation.

$$
d=\left(\lambda_{\max }-n\right) /(n-1) .
$$

The maximum eigenvalue method is used to estimate the weights of criteria originally. And, the Saaty's consistency function is given by the following equation.

$$
f(\hat{w})=\frac{1}{n \cdot(n-1)} \sum_{i=1}^{n} \sum_{j=1}^{n}\left(a_{i j} \cdot \frac{\hat{w}_{j}}{\hat{w}_{i}}-1\right) .
$$

If $\hat{w}=\left(\hat{w}_{1}, \ldots, \hat{w}_{n}\right)^{\top}$ is the eigenvector corresponding the maximum eigenvalue, the value of this function is $d$. This function assumes that the estimated weight vector is near on the eigenvector corresponding the maximum eigenvalue (we call the maximum eigenvector). In other words, this function represents the distance between the estimated weight vector and the maximum eigenvector. To calculate a consistency degree of the geometric mean method, this Saaty's function is also utilized. Though the geometric mean method has a proper consistency function [1],[2]; it does not be used generally. For measurement of the consistency, there are not only those methods but also the general mean methods which were proposed in [4]. The estimated weights of this general mean methods are given by the following:

$$
\hat{w}_{i}=\left(\frac{1}{n} \sum_{j=1}^{n} a_{i j}^{r}\right)^{1 / r}, \quad r \neq 0 .
$$

In this paper, we assume that the matrix of pairwise comparisons is reciprocal. Therefore this weight vector is the optimal solution of the next least squares problem.

$$
\min _{w_{i}} \sum_{i} \sum_{j} \frac{1}{2 n^{2}}\left(\left(\sqrt{a_{i j}} w_{j}\right)^{-r}-\left(\sqrt{a_{j i}} w_{i}\right)^{-r}\right)^{2}
$$


s.t. $\quad\left(\frac{1}{n} \sum_{i=1}^{n} w_{i}^{-r}\right)^{-\frac{1}{r}}=1$.

When $r=1$, this weight is the arithmetic mean of the row of the matrix, and when $r=-1$, it is the harmonic mean. In addition, it becomes the maximum value of the row elements if $r \rightarrow \infty$ and becomes the minimum value if $r \rightarrow-\infty$. The minimum value $h$ of the objective function (the residual sum of squares) is given by the next equation.

$$
h=\frac{1}{\sum_{i=1}^{n} \frac{1}{\sum_{j=1}^{n} a_{i j}^{r}}}-1 .
$$

Since the function (3) is the least squares form, this value can be used a measure of consistency. For example, when the matrix is consistent this value is zero and the estimated weights are the true weights.

\section{An error in a specific pair of elements}

It is difficult that it detects the statistical distribution of errors including in the comparison matrix. In this section, we discuss the estimated weights when pairwise comparison matrix includes a fluctuation in a specific pair of elements. We assume that the $(i, j)$ element of the comparison matrix is $\delta w_{i} / w_{j}$ and the $(j, i)$ element is $w_{j} /\left(\delta w_{i}\right)$ for some positive number $\delta$.

\section{A. The case of the geometric mean method}

Since an error is included in the elements $(i, j)$ and $(j, i)$, the geometric means are given by the following.

$$
\begin{aligned}
x_{i} & =\sqrt[n]{a_{i 1} a_{i 2} \cdots a_{i n}}=\frac{\sqrt[n]{\delta} \cdot w_{i}}{\sqrt[n]{w_{1} \cdot w_{2} \cdots w_{n}}}, \\
x_{j} & =\frac{1}{\sqrt[n]{\delta}} \frac{w_{j}}{\sqrt[n]{w_{1} \cdot w_{2} \cdots w_{n}}}, \\
x_{k} & =\frac{w_{k}}{\sqrt[n]{w_{1} \cdot w_{2} \cdots w_{n}}}, \quad k \neq i \text { and } k \neq j .
\end{aligned}
$$

\section{B. The case of the eigenvalue method}

In this case, the following simultaneous equations are formed for an eigenvector. Here the element with the fluctuation sets to $a_{12}$ without loss of generality.

$$
\begin{aligned}
& A x=\lambda \boldsymbol{x} \\
& x_{1}+\frac{w_{1}}{w_{2}} \delta x_{2}+w_{1} \sum_{\ell=3}^{n} \frac{x_{\ell}}{w_{\ell}}=\lambda x_{1}, \\
& \frac{w_{2}}{w_{1}} \frac{x_{1}}{\delta}+x_{2}+w_{2} \sum_{\ell=3}^{n} \frac{x_{\ell}}{w_{\ell}}=\lambda x_{2}, \\
& \frac{w_{k}}{w_{1}} x_{1}+\frac{w_{k}}{w_{2}} x_{2}+w_{k} \sum_{\ell=3}^{n} \frac{x_{\ell}}{w_{\ell}}=\lambda x_{k}, \quad k=3, \cdots, n .
\end{aligned}
$$

From the equation (9), $x_{k} \propto w_{k}, k \geq 3$. We will eliminate $x_{1}, x_{2}$ from the above equations, then we obtain a polynomial function of $\lambda$.

$$
\lambda^{3}-n \lambda^{2}-(n-2)\left(\frac{1}{\delta}+\delta-2\right)=0 .
$$


Thus, the solution of the above polynomial function is

$$
\begin{aligned}
\lambda=\frac{n}{3}+\frac{2^{\frac{1}{3}} n^{2}}{3\left(27 A+2 n^{3}+3 \sqrt{3} \sqrt{A}\right.} \sqrt{\left.27 A+4 n^{3}\right)^{\frac{1}{3}}} & \\
& +\frac{3\left(27 A+2 n^{3}+3 \sqrt{3} \sqrt{A} \sqrt{27 A+4 n^{3}}\right)^{\frac{1}{3}}}{2^{\frac{1}{3}} n^{2}},
\end{aligned}
$$

where $A=(n-2)\left(\frac{1}{\delta}+\delta-2\right)$.

Therefore, we have

$$
\begin{aligned}
& x_{1}=\frac{(\lambda-1+\delta)(n-2)}{\lambda(\lambda-2)} \alpha w_{1}, \\
& x_{2}=\frac{\left(\lambda-1+\frac{1}{\delta}\right)(n-2)}{\lambda(\lambda-2)} \alpha w_{2}, \\
& x_{k}=\alpha w_{k}, \quad k \geq 3 .
\end{aligned}
$$

\section{The case of the general mean method}

The general means of the row are given by the following.

$$
x_{k}=\left(\frac{1}{n} \sum_{\ell=1}^{n} a_{k \ell}^{r}\right)^{\frac{1}{r}}, \quad k=1, \ldots, n .
$$

Therefore,

$$
\begin{aligned}
& x_{i}=\left(\frac{1}{n}\left\{\sum_{\ell=1, \ell \neq j}^{n} \frac{1}{w_{\ell}^{r}}+\frac{\delta^{r}}{w_{j}^{r}}\right\}\right)^{\frac{1}{r}} w_{i}, \\
& x_{j}=\left(\frac{1}{n}\left\{\sum_{\ell=1, \ell \neq i}^{n} \frac{1}{w_{\ell}^{r}}+\frac{1}{w_{i}^{r} \delta^{r}}\right\}\right)^{\frac{1}{r}} w_{j}, \\
& x_{k}=\left(\frac{1}{n} \sum_{\ell=1}^{n} \frac{1}{w_{\ell}^{r}}\right)^{\frac{1}{r}} w_{k}, \quad k \neq i \text { and } k \neq j .
\end{aligned}
$$

Now, the distance between the true weights and the estimated weights is calculated as follows. The $x_{i}$ must be normalized to which the sum is 1 . Therefore,

$$
\begin{aligned}
& \sum_{k=1}^{n}\left(\hat{w}_{k}-w_{k}\right)^{2}=\sum_{k=1}^{n}\left(x_{k} / S-w_{k}\right)^{2} . \\
& \quad=\left((S-1)^{2} \sum_{k=1}^{n} w_{k}^{2}+(p-1)(p+1-2 S) w_{i}^{2}+(q-1)(q+1-2 S) w_{j .}^{2}\right) / S^{2},
\end{aligned}
$$

where $p=x_{i} / w_{i}, q=x_{j} / w_{j}, S=\sum_{i=1}^{n} x_{i}$.

If $\delta$ is a large number, the distance of the geometric mean method is smaller than the eigenvalue methods one, because $\lambda$ of the eigenvalue method has $O\left(\delta^{\frac{1}{3}}\right)$.

Theorem 1. If $\delta$ is a large number and the number of criteria is greater than 4 , the estimated weights of the geometric method are close to the true weights than the weights of the eigenvalue methods. 
Proof. From the equations (4) - (6), the weights of the geometric mean method are given by

$$
x_{i}=\sqrt[n]{\delta} \cdot C_{1}, \quad x_{j}=C_{2} / \sqrt[n]{\delta}, \quad x_{k}=C_{k}, \quad k \neq i \text { and } k \neq j,
$$

where $C_{1}, C_{2}, \ldots, C_{n}$ are constant numbers. Since $\lambda$ of the eigenvalue method is $O\left(\delta^{\frac{1}{3}}\right)$, the weights of eigenvalue method are

$$
x_{i}=\sqrt[3]{\delta} \cdot \hat{C}_{1}, \quad x_{j}=\hat{C}_{2} / \sqrt[3]{\delta}, \quad x_{k}=\hat{C}_{k}, \quad k \neq i \text { and } k \neq j .
$$

And form the equation (16), the proof is completed.

In general, the estimated weights of the geometric method are not always better than the weights of the general mean methods. For instance, if the weights are estimated by the general mean method with $r=-1$ (harmonic mean), $w_{j}$ is close to zero and the other $w_{k}$ are slight bigger than the true weights.

Theorem 2. If $\delta$ is a large number, the estimated weights of the geometric method are not always close to the true weights than the weights of the general mean methods with $-\infty<r<0$.

Proof. If $\delta$ is a large number and $r$ is negative, from the equations (13)-(15)

$$
\begin{aligned}
& x_{i} \approx\left(\frac{1}{n} \sum_{\ell=1, \ell \neq i}^{n} \frac{1}{w_{\ell}}\right)^{\frac{1}{r}} w_{i}, \\
& x_{j} \approx 0, \\
& x_{k}=\left(\frac{1}{n} \sum_{\ell=1}^{n} \frac{1}{w_{\ell}^{r}}\right)^{\frac{1}{r}} w_{k}, \quad k \neq i \text { and } k \neq j .
\end{aligned}
$$

On the other hand, the geometric means are

$$
x_{i} \approx 1, \quad x_{j} \approx 0, \quad x_{k} \approx 0, \quad k \neq i \text { and } k \neq j .
$$

Therefore, the weights of the general mean method are close to the true weights than the weights of the geometric mean method.

\section{The distance between the weights}

In the previous section, the estimated weights of the general mean methods are close to the true weight than the geometric mean and/or the maximum eigenvector in the suitable situation. In the geometric mean method, the distribution of the errors in the matrix is the $\log$ normal distribution. And in the case of the general mean method, if the value of $\left(\sqrt{a_{i j}} w_{i}\right)^{-r}-\left(\sqrt{a_{j i}} w_{j}\right)^{-r}$ is according to the normal distribution, the weight vector generated by the general mean method is the best least squares solution. Therefore, the residual sum of squares also depend on the original errors. So, we shall study the characteristics of the distances between the estimated weights and the original weights.

At first, we calculate the distance between the weights in which the error distribution is the log normal distribution. Figure 1. shows the distances of the weights of the general mean method at $r$. The plot curves in Figure 1. show the distance when the standard deviation of the normal distribution is $3.0,1.0,0.5,0.3$, 0.1 from an upside. In the simulation, the original weight is generated by a random number in $[0.1,1)$ and normalized. In the figure, the distance between the geometric mean and the true weight is the value at $r=0$ because the general mean with $r=0$ is the geometric mean. From the logarithm least squares, the geometric mean is the best least square estimator in which the error distribution is the log normal. Nevertheless, from Figure 1., the general mean method is not bad in comparison with the geometric mean method. 


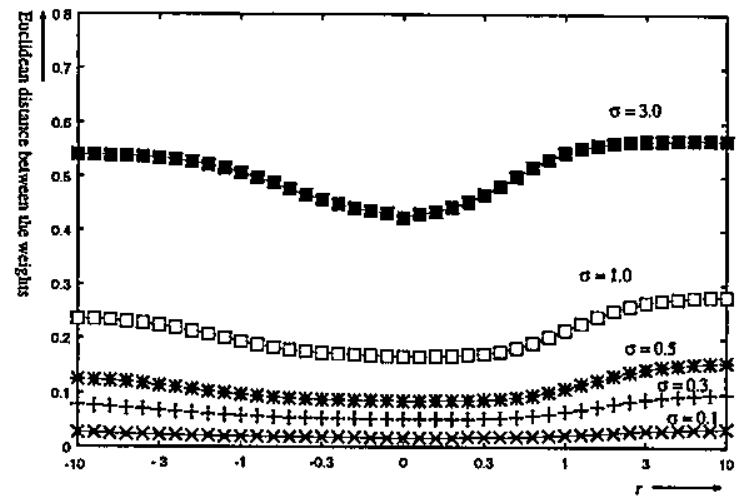

Figure 1 : The log normal distribution (5 criteria, 500 simulations)

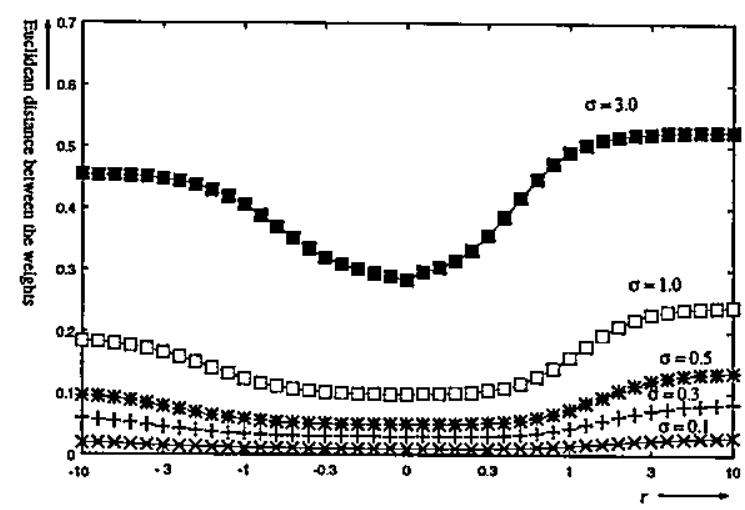

Figure 2 : The log normal distribution (10 criteria, 500 simulations)

And we calculate the distances when the tail of error distribution is heavy. Figure 3, 4 and 5 show the distances at $r$ when the error distribution is given by the next routine.

1. Generate random numbers $R_{k}$ according to the one of the next distributions.

Type A : $f(x)=\frac{1}{\pi \cdot\left(x^{2}+1\right)}$,

Type B : $f(x)=2\left(\frac{1}{\exp (x)+\exp (-x)}\right)^{2}$,

Type C : $f(x)=\frac{1}{\sqrt{2 \pi} \sigma} \exp \left(\frac{-x^{2}}{2 \sigma^{2}}\right)$.

2. Set $a_{i j}=\frac{w_{i}}{w_{j}} \exp \left(R_{k}^{n}\right)$ for a given number $n$.

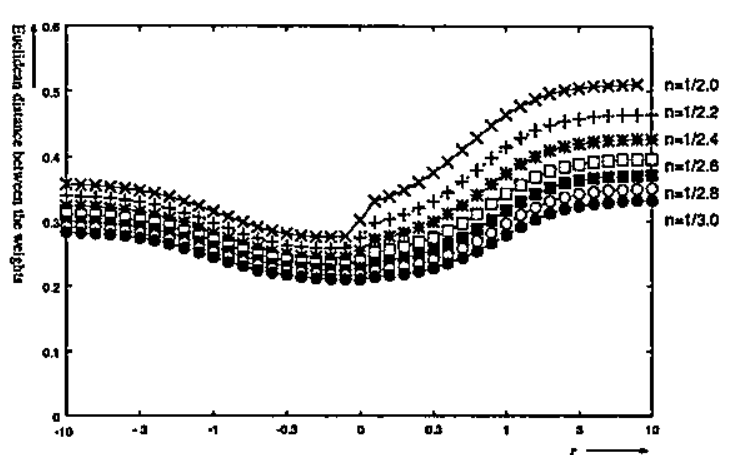

Figure 3 : Type A:

$n=1 / 2.0,1 / 2.2,1 / 2.4,1 / 2.6,1 / 2.8,1 / 3.0$

( 5 criteria, 500 simulations)

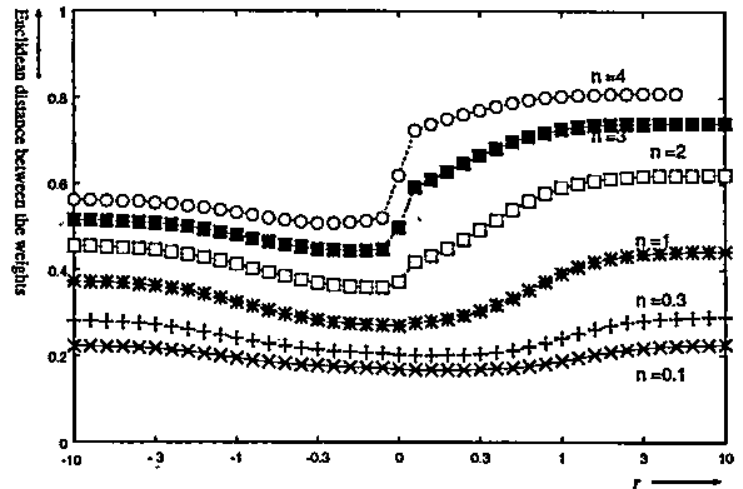

Figure 4 : Type B:

$n=0.1,0.3,1,2,3,4$

( 5 criteria, 500 simulations) 


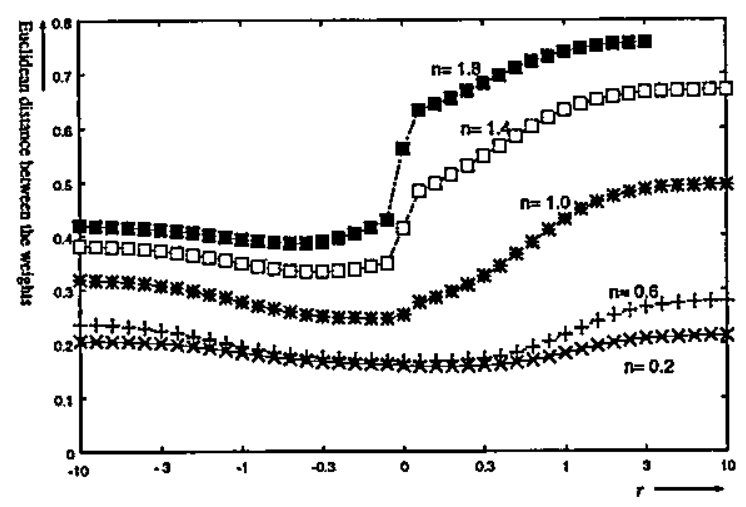

Figure 5 : Type C:

$n=0.2,0.6,1.0,1.4,1.8$

(5 criteria, 500 simulations)

Since the variances of those distributions are large, the general mean method with $r<0$ is better than the geometric mean method. But, if $r \geq 0$, the geometric mean is the best.

\section{Conclusion}

From these results, there are some advantages of the general mean method .

1. The parameter $r$ should set to a negative value in the case of the distribution with the heavy tail.

2. The geometric mean method is not always close to the true weight.

3. If the parameter $r$ is a positive number, the estimated weight is far to the true weight.

4. The harmonic mean method which is the general mean method with $r=-1$ is useful.

\section{Reference}

[1] Saaty,T.L. (1980) The Analytic Hierarchy Process, McGraw-Hill.

[2] Saaty,T.L. and Vargas,G.V.(1984) Comparison of Eigenvalue, Logarithmic Least Squares and Least Squares Methods in Estimating Ratios, Mathematical Modelling, Vol.5, pp.309-324.

[3] Saaty,T.L. (199) Eigenvector and logarithmic least squares, European Journal of OR, Vol.48, pp.156160.

[4] Kato, Y., and Ozawa, M.(1998) The harmonic method on AHP, the abstracts in the 58th Quality Control conference (in Japanese), pp.59-62.

[5] Nishina,K. and Shibayama, T.(1992) A comparison of the Eigenvector method and the logarithmic least squares method in paired comparisons, Quality(JSQC), Vol.22, pp.115-123. 